\begin{tabular}{|c|l|}
\hline Title & Spectroscopic study of mutual solubilities of water and benzene at high temperatures and pressures \\
\hline Author(s) & Jin, Y usuke; Ikawa, Shun-ichi \\
\hline Citation & $\begin{array}{l}\text { The Journal of Chemical Physics, 122(24509), 1-7 } \\
\text { https://doi.org/10.1063/1.1829254 }\end{array}$ \\
\hline Issue Date & 2004 12-22 \\
\hline Doc URL & http://hdl.handle.net/2115/1394 \\
\hline Rights & Copyright $\odot 2004$ A merican Institute of Physics \\
\hline Type & article \\
\hline File Information & JCP122-24509.pdf \\
\hline
\end{tabular}

Instructions for use 


\title{
Spectroscopic study of mutual solubilities of water and benzene at high temperatures and pressures
}

\author{
Yusuke Jin and Shun-ichi Ikawa \\ Division of Chemistry, Graduate School of Science, Hokkaido University, Sapporo, 060-0810, Japan
}

(Received 24 June 2004; accepted 14 October 2004; published online 22 December 2004)

\begin{abstract}
Near-infrared and ultraviolet absorption of water-benzene mixtures has been measured at temperatures and pressures in the ranges of 323-673 $\mathrm{K}$ and 50-400 bar, respectively. Concentrations of water and benzene in both the water-rich phase and the benzene-rich phase of the mixtures were obtained from absorption intensities of near-infrared bands of water and benzene and ultraviolet bands of benzene. Mutual solubilities in molar fractions increase remarkably with increasing temperature at pressures in the two-liquid-phase coexistence region, and are consistent with previously reported values. It proves that the solubility of benzene in water is an order of magnitude smaller than that of water in benzene throughout the two-phase region. In addition, it is found that effect of pressure on the solubilities is opposite between water in benzene and benzene in water. These solubility properties are discussed on the basis of a cavity-based solvation model. It is suggested that the asymmetry in the mutual solubility and the opposite direction of the pressure effect are caused by difference in molecular size and difference in thermal compressibility, respectively, between water and benzene. (C) 2005 American Institute of Physics.
\end{abstract}

[DOI: $10.1063 / 1.1829254]$

\section{INTRODUCTION}

Water and hydrocarbons are almost immiscible or only poorly miscible with each other at room temperature. However, their mutual solubilities are of fundamental importance for the study on the water-hydrocarbon interaction, which is concerned with a wide variety of naturally occurring phenomena. The solubility data are particularly important to characterize hydrophobic nature of various segments of proteins, which plays an important role in formation and stabilization of the biologically active structures. ${ }^{1-3}$ The solubility data are also useful to assessment and suitable treatment of water pollution by hydrocarbons, which leads to air pollution also. ${ }^{4,5}$ So far a great number of experimental data of mutual solubilities for water-hydrocarbon systems have been accumulated. ${ }^{6-8}$ Most of the data are of ambient condition or not far from it, but solubilities at high temperatures and pressures have scarcely been reported. Recently, solubilities of aqueous systems at high temperatures have been paid much attention. These data are required for pollution assessment of high-temperature water discharged from laboratories and industrial plants, ${ }^{9-13}$ and moreover, for designing an environment protecting technology that uses high-temperature water in the sub- to supercritical condition as a reaction medium for destruction of toxic waste chemicals. ${ }^{14-17}$ In addition, the high-temperature solubility data are indispensable to designs, operations and maintenance of plants for oil refineries and petrochemical industry. ${ }^{9,18-20}$

Nevertheless, mutual solubilities of water and hydrocarbons at high temperatures are scarce and considerably scatter even for the water-benzene system that has been most extensively studied among others. ${ }^{7}$ This is due to difficulty in measurements of solubilities at high temperatures and pressures. Usually a small amount of mixtures equilibrated in a high temperature-pressure vessel is sampled and transferred to analytical instruments such as gas chromatographs and Karl Fisher titration instruments for composition analysis. The transferring of a sample without disturbing equilibrium and/or without composition change is rather difficult, particularly at high temperatures and pressures. ${ }^{10,21}$ Therefore most of the solubility data of the water-benzene system have been limited at the three-phase equilibrium pressures, $, 10,13,21$ and a few other pressures at higher temperatures. ${ }^{22-25}$ In addition, effect of pressure on the mutual solubilities has not been fully understood, in contrast to the temperature effect, namely the significant increase in the mutual solubilities with increasing temperature.

In this context, we have attempted to measure the mutual solubilities of water and benzene at high temperatures in the two-liquid-phase coexistence region using in situ spectroscopy. This method enables us to determine concentrations of water and benzene in both the water-rich phase and the benzene-rich phase of the high-temperature mixtures without disturbing the equilibrium. Recently, in situ infrared and Raman spectroscopy has been successfully applied to studies of molecular-level structure of high-temperature aqueous mixtures. It revealed how the rotational motion and the hydrogen-bonded association of water molecules in the mixtures vary with temperature and pressure. ${ }^{26-30}$ In addition, it has been shown that a concept of $\pi$-hydrogen bond is still valid for describing intermolecular interaction between water and aromatic ring at temperatures as high as $523 \mathrm{~K}$ under pressure. $^{28,31}$ Moreover, it is only the in situ measurements that can directly provide volumetric concentrations for each phase of the two coexisting liquid-phases. Using the volumetric concentrations obtained from infrared and nearinfrared measurements, we could find the anomalous volumetric expansion on the mixing of water and hydrocarbons in the vicinity of the critical region. ${ }^{32,33}$ 


\section{EXPERIMENTAL METHODS AND RESULTS}

A high-temperature-pressure cell used has been described previously. ${ }^{27,33}$ Distilled and deionized water with electric conductivity of less than $0.2 \mu \mathrm{S}^{-1}$ was used, and spectroscopic grade benzene from Nacalai Tesque (Japan) was used as received. Near-infrared spectra in the 5500$7800 \mathrm{~cm}^{-1}$ range were measured with a Perkin-Elmer System 2000 Fourier-transform spectrometer equipped with a near-infrared source and a near-infrared detector. Spectral measurements were performed with $2 \mathrm{~cm}^{-1}$ resolution at sample temperatures in the $423-673 \mathrm{~K}$ range and pressures in the 50-400 bar range. Ultraviolet spectra in the 220-300 $\mathrm{nm}\left[(4.5-3.3) \times 10^{6} \mathrm{~cm}^{-1}\right]$ range were measured with a JASCO V-550 spectrometer with $1 \mathrm{~nm}$ resolution at temperatures and pressures in the ranges of $323-498 \mathrm{~K}$ and $50-400$ bar, respectively. The optical path length of the cell was 1.46 $\mathrm{mm}$ for the near-infrared measurements and $0.34 \mathrm{~mm}$ for the ultraviolet measurements. The sample temperature was measured with a Chromel-Alumel thermocouple inserted directly into the sample fluid and controlled by a digital controller with an accuracy of $\pm 1.0 \mathrm{~K}$. The sample pressure was measured with a pressure transducer of a semiconductor strain gage and controlled within \pm 0.5 bar. Each of the experimental temperature-pressure points was attained at a slow enough rate, and the phase equilibrium of the sample fluid was confirmed by the spectrum that remained unchanged for at least an hour.

\section{A. Observed near-infrared spectra}

Figure 1 shows observed near-infrared spectra of the water-rich phase (a) and the benzene-rich phase (b) of the water-benzene mixtures at a constant pressure of 200 bar. Absorption at around $7100 \mathrm{~cm}^{-1}$ is assigned to the $\mathrm{OH}-$ stretching overtone transitions of water and that at around $6000 \mathrm{~cm}^{-1}$ to the $\mathrm{CH}$-stretching overtone transitions of benzene. The shoulder at $7400 \mathrm{~cm}^{-1}$ is attributed to the R-branch of the rotational structure of water molecules, while that at $6800 \mathrm{~cm}^{-1}$ is mainly attributed to hydrogenbonded small clusters. ${ }^{27}$ The spectra of the water-rich phase (a) were described in detail in the previous paper. ${ }^{33}$ For the spectra of the benzene-rich phase (b), the benzene absorption decreases monotonously with increasing temperature. On the other hand, the absorption of water as a solute initially increases with increasing temperature up to $573 \mathrm{~K}$, and then decreases at higher temperatures. In addition, even the rotational fine structures can be seen at the higher temperatures. This means that significant proportion of the water molecules in the benzene-rich phase rotate quite freely, where collisions with surrounding molecules are not frequent enough to completely suppress the sharp peaks of the fine structure.

\section{B. Observed ultraviolet spectra}

Near infrared absorption of benzene in water at temperatures in the two-phase region is very weak due to the poor solubility of benzene in water, and is difficult to provide reliable concentration. Then we have measured ultraviolet absorption of benzene, which is attributed to a $\pi-\pi^{*}$ transition. This absorption has been used to measure concentra-

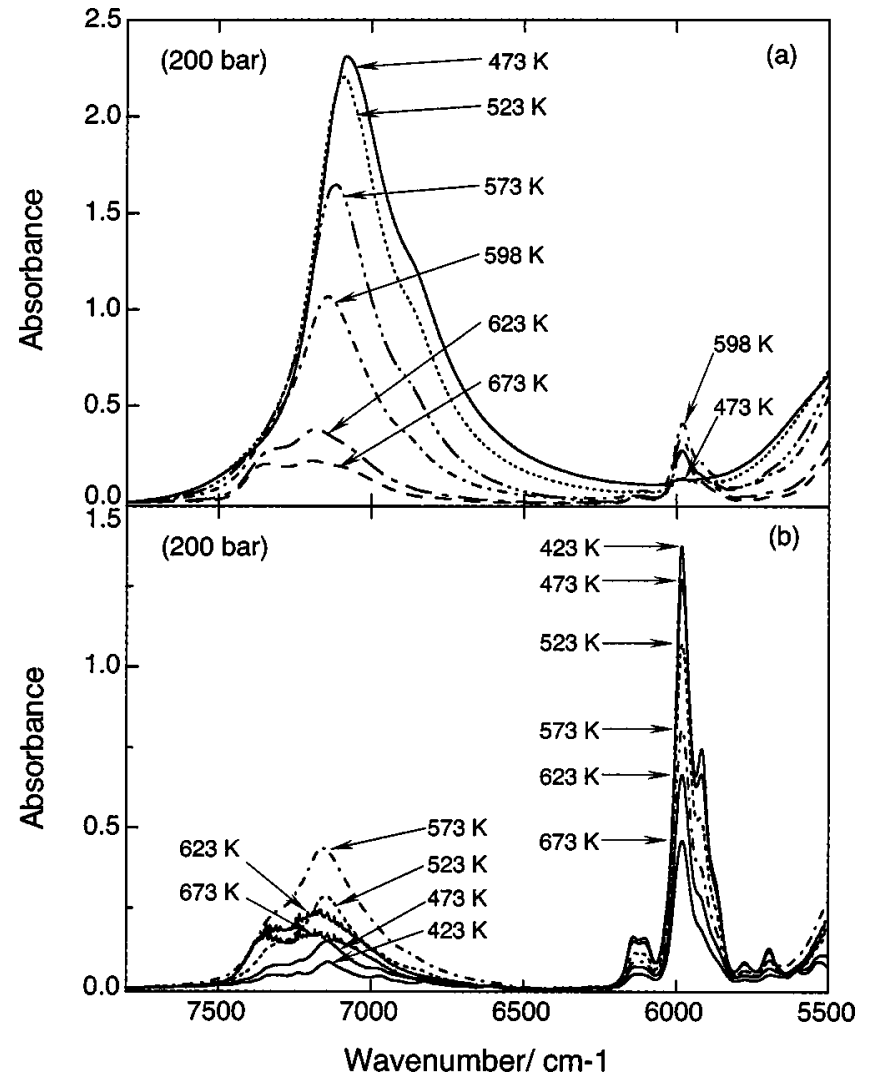

FIG. 1. Near infrared spectra of (a) the water-rich phase and (b) the benzene-rich phase of the water-benzene mixtures at various temperatures and at a constant pressure of 200 bar.

tions of benzene in water at high pressures. ${ }^{34,35}$ However, measurements at high temperatures and pressures have scarcely been reported.

Figure 2(a) displays examples of observed ultraviolet absorption of benzene in the water-rich phase at various temperatures and at a constant pressure of 150 bar. Several peaks with roughly the same interval seen in the spectra are the vibrational progressions originated from the totally symmetric ring-breathing mode of benzene. The positions of these peaks are consistent with those observed in a low-resolution gas-phase spectrum, ${ }^{36,37}$ though slightly shift to longer wavelength. The gas-phase spectra has been fully analyzed. The somewhat long progression is caused by the increase in all the $\mathrm{C}-\mathrm{C}$ bond lengths from $1.397 \AA$ (ground state) to 1.434 $\AA$ (excited state), and the interval of the progression, 923 $\mathrm{cm}^{-1}$ (gas phase), gives a vibrational frequency of the breathing mode in the excited state. This frequency is distinctly smaller than that in the ground state, $992 \mathrm{~cm}^{-1}$ (gas phase), and consistent with the longer bond length in the excited state. The peaks of the spectra shown in Fig. 2 are very broad due to collapse of the rotational fine structures and their intervals could not be estimated with a precision comparable to the gas-phase spectra. Nevertheless, the average interval of roughly $880 \mathrm{~cm}^{-1}$ is not far from the abovementioned gas phase value. This fact suggests that the ex- 


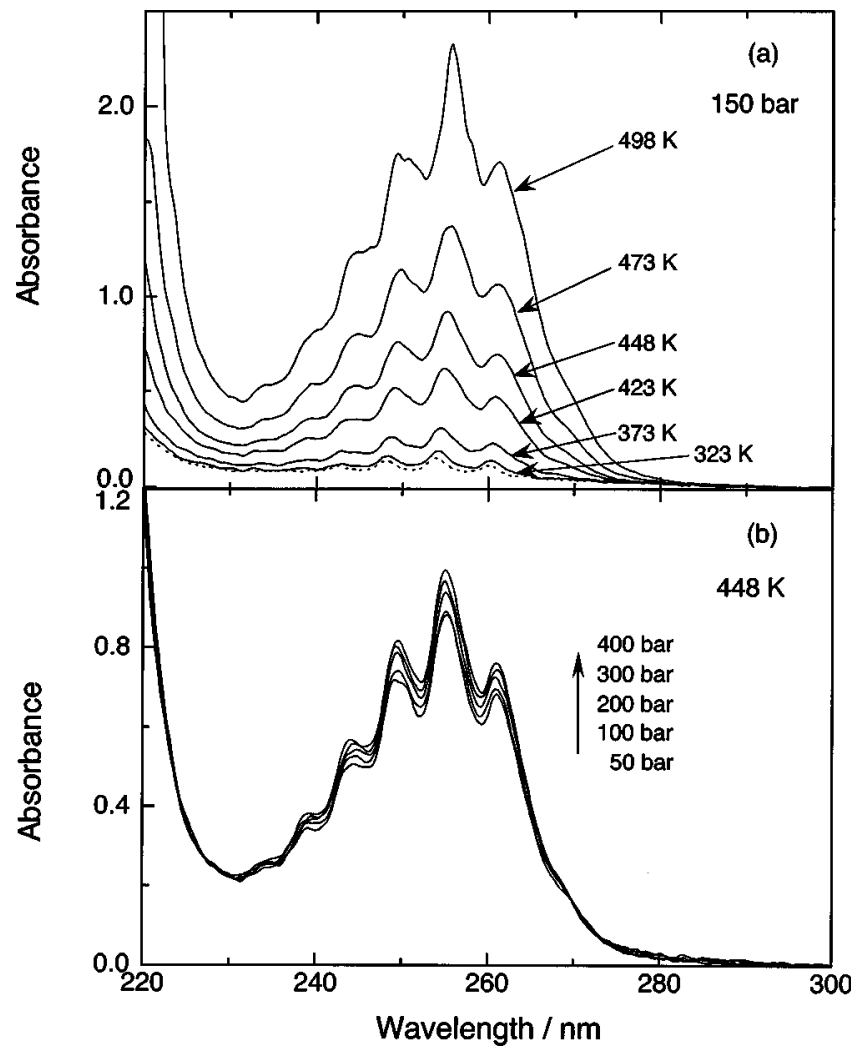

FIG. 2. Ultraviolet absorption of benzene in water (a) at various temperatures and at a constant pressure of 150 bar and (b) at various pressures and at a constant temperature of $448 \mathrm{~K}$.

cited state of benzene and its vibrational structure does not significantly change on transfer from the gas phase into the high-temperature water. The remarkable increase in the absorbance with increasing temperature is due to increase in benzene concentration. The absorption profile is approximately the same at different temperatures, although a faint fine structure can be seen at the higher temperatures. Each of the peaks exhibits a very small shift to longer wavelength with rising temperature (about $0.015 \mathrm{~nm} / \mathrm{K}$ ).

Figure 2(b) shows pressure dependence of the absorption at constant temperature of $448 \mathrm{~K}$. The absorption intensities increase only slightly with increasing pressure from 50 to $400 \mathrm{bar}$, in contrast to the remarkable increase with rising temperature shown in Fig. 2(a).

\section{DISCUSSION}

\section{A. Estimation of concentrations}

Concentrations of water and benzene were obtained from observed near-infrared band intensities in the same way as in the previous work. ${ }^{33}$ Integration ranges of the absorption were $6200-7800 \mathrm{~cm}^{-1}$ and $5824-6064 \mathrm{~cm}^{-1}$ for the water and benzene bands, respectively. The base lines were drawn by connecting the signal levels of the both ends of the integration, to minimize effect of band overlapping.

To estimate the concentrations, molar absorption intensities are required. Allowing for the fact that the molar absorption intensities of water vary with the hydrogen bonding state, we have proposed the following expression for the molar absorption intensity: ${ }^{38}$

$$
\begin{aligned}
\left(A^{m} / \mathrm{cm} \mathrm{mol}^{-1}\right)= & 7.4108 \times 10^{7}-2.1260 \times 10^{4}\left(\bar{\nu} / \mathrm{cm}^{-1}\right) \\
& +1.5317\left(\bar{\nu} / \mathrm{cm}^{-1}\right)^{2},
\end{aligned}
$$

where, $A^{m}$ and $\bar{\nu}$ denote the molar absorption intensity and the first band moment, respectively. This expression has been obtained from an empirical relationship between the molar absorption intensity and the first band moment of neat water at various temperatures and pressures, using literature data of the water density. ${ }^{39}$ The first band moment can be obtained from a normalized absorption profile on condition that the absorption does not significantly overlap with other absorption. Then the water concentration in the mixtures is given by,

$$
\left(C_{W} / \mathrm{moll}^{-1}\right)=10^{3} \times\left(A_{\text {obs }} / \mathrm{cm}^{-2}\right) /\left(A^{m} / \mathrm{cm} \mathrm{mol}^{-1}\right) .
$$

The molar absorption intensity of benzene band was assumed to be given by that of neat benzene at the same temperature and pressure. This method has been supported by the fact that the position and the profile of the overtone absorption of benzene exhibit little change on going from neat liquid to the aqueous mixtures, indicating the transition is insensitive to the molecular environmental change. ${ }^{33}$

Ultraviolet absorption of benzene was integrated over the 230-290 nm range, where the baseline was taken as a straight line connecting the both ends of the absorption. Concentrations of benzene were estimated from ratios of the integrated intensities to that measured at $298 \mathrm{~K}$ and $1 \mathrm{bar}$, at which solubility of benzene in water has been extensively studied and a value of $0.0226 \mathrm{~mol} / \mathrm{l}$ has been recommended. ${ }^{7}$ Since water is completely transparent in the ultraviolet region, we can not obtain water concentrations in the same solutions from the ultraviolet measurements. Therefore, we have estimated water concentrations on the assumption that the molar volumes of water and benzene are additive in the temperature range of 323-498 K. Then the water concentration in the water-rich phase is given by

$$
C_{W}=C_{W}^{0}-C_{B} \frac{v_{B}^{0}}{v_{W}^{0}},
$$

where $v_{W}^{0}$ and $v_{B}^{0}$ are molar volumes of neat water and neat benzene, respectively, which have been calculated from literature data. ${ }^{39,40}$ The above-mentioned temperature range is far from the critical region where the mixtures exhibit anomalous volumetric behavior, ${ }^{33}$ and Eq. (3) is expected to provide a good approximation as for ordinary liquid mixtures.

The mutual solubilities in molar fractions are calculated from the concentrations obtained above. The resulting values at a constant pressure of 200 bar are plotted against temperature in Fig. 3, and are in good agreement with previously reported values ${ }^{22-25}$ which are indicated by open symbols. At temperatures higher than the critical solution temperature, $570 \mathrm{~K},{ }^{23}$ the mixtures form the homogeneous phase at any mixing ratio. Therefore, the molar fractions obtained in that region are not concerned with the mutual solubility but 


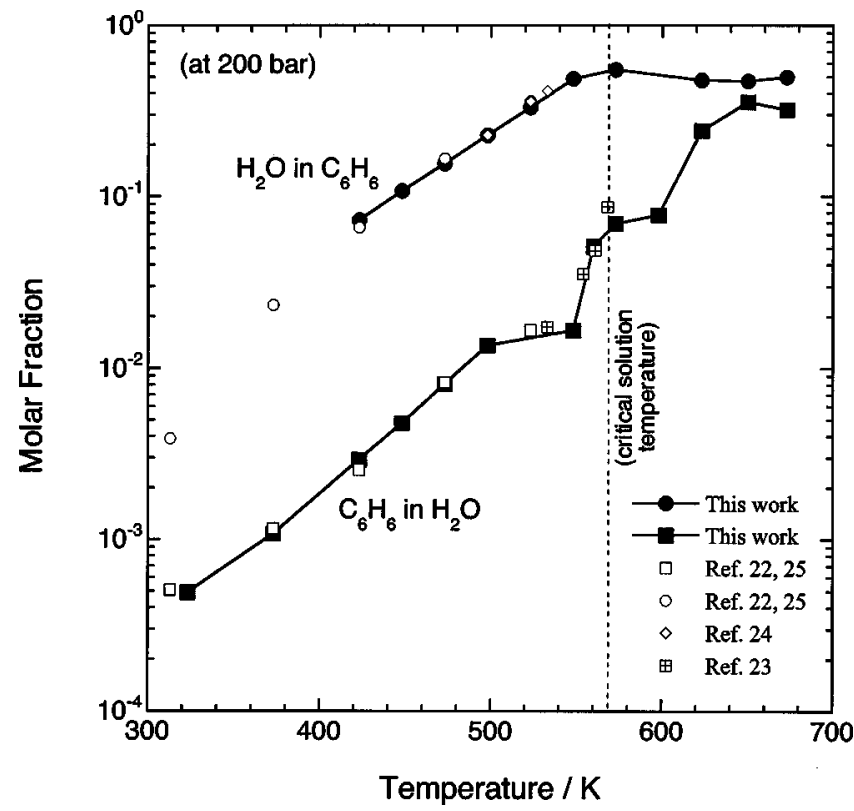

FIG. 3. Mutual solubilities in molar fraction for water-benzene mixtures plotted against temperature at a constant pressure of 200 bar.

should be dependent on experimental condition such as an initial fluid composition in the cell and a way to attain the experimental temperature-pressure points. In the two-phase region, at temperatures below $570 \mathrm{~K}$, both the solubilities of water in benzene and benzene in water remarkably increase with increasing temperature. In addition, it is obvious that the solubility of benzene in water is an order of magnitude smaller than that of water in benzene. This asymmetry of the mutual solubility is consistent with tendency for waterhydrocarbon systems ${ }^{41}$ at room temperature. Figure 4 shows pressure dependence of the mutual solubilities at several temperatures. Although the pressure dependence is very small as compared with the temperature dependence, it should be noticed that the solubility of benzene in water and that of water in benzene exhibit opposite pressure dependence. The former increases whereas the latter decreases, with increasing pressure at a constant temperature. This result on the opposite pressure dependence is consistent with previous observations. ${ }^{9,25,42}$ A systematic study of the pressure dependence of the mutual solubilities at high temperatures, however, has never been reported so far to our knowledge, though the pressure dependence of the solubilities at around room temperature has been studied in detail..$^{34,35}$

To discuss further the solubility properties, it is worthwhile to mention experimental errors in the present results. Reproducibility of the integrated band intensities was quite good and the uncertainty was less than a few percent. However, uncertainty of the absolute values of the water concentrations estimated by Eq. (2) may be somewhat larger, because of use of the empirical relationship Eq. (1) to estimate the molar absorption intensity. Nevertheless, it may be less than $20 \%$ judging from comparison with previous values cited in Fig. 3. On the other hand, relative variations of the concentrations with temperature and pressure shown in Fig. 4 will have much higher reliability than their absolute values. The relative values of the concentrations are mostly given by
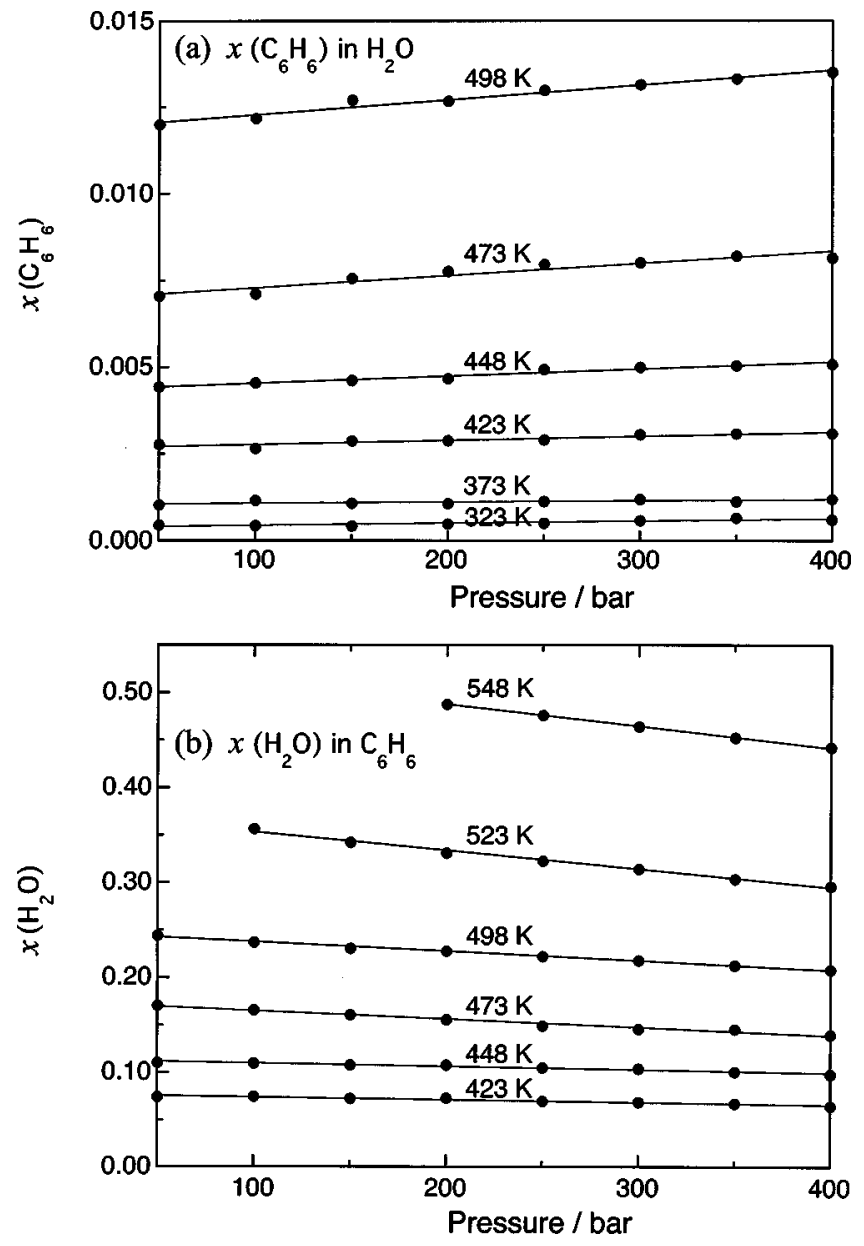

FIG. 4. Pressure-temperature dependence of the solubilities in molar fraction for (a) benzene in water and (b) water in benzene.

the relative integrated band intensities, and the concentration variations should be determined within a few percent uncertainty. In addition, the pressure dependence seen in Fig. 4 is, at least qualitatively, consistent with the previous observations. ${ }^{9,25,42}$ Therefore, the temperature-pressure-depending tendency of the mutual solubilities shown in Figs. 3 and 4 is quite certain and worth while to discuss further.

To understand the asymmetric features of the magnitude and the pressure dependence of the mutual solubilities for the water-benzene system from a molecular viewpoint, we will discuss the mutual solubilities on the basis of a cavity-based model in the next section.

\section{B. Solubility from a molecular viewpoint}

Hydrophobic hydration has been one of the most important subjects of solution chemistry and biophysical chemistry. ${ }^{43-45}$ To reveal the nature of the hydrophobic hydration, solubilities of hydrocarbons in water have been used as basic data. One intriguing fact is that aromatic hydrocarbons in general are much more soluble than aliphatic hydrocarbons in water. ${ }^{41}$ This is naturally attributed to $\pi$ electrons of the aromatic ring. However, a mechanism of the $\pi$ electrons action is still a question in controversy. One plausible explanation is that the $\pi$-hydrogen bonding with water is responsible for the higher solubility in water and then the 
smaller hydration free energy of the aromatic hydrocarbons. ${ }^{46,47}$ The $\pi$-hydrogen bonding is an important concept to understand gas-phase structure of water-benzene complexes $^{48,49}$ and various spectroscopic behaviors of the water-benzene mixtures. ${ }^{28,31,50,51}$ Very recently, on the other hand, Graziano ${ }^{52}$ has proposed an important role of the van der Waals interaction that is strengthened by a larger polarizability density of the aromatic ring as compared with aliphatic hydrocarbons. He has suggested that the formation of the $\pi$-hydrogen bonds between water and benzene is largely an enthalpy-entropy compensating process that does not affect the hydration free energy. Another basic problem is to what extent the water-water hydrogen bonding affects the hydrophobic hydration. Recently, Ikeguchi et al. ${ }^{53}$ have indicated on the basis of Monte Carlo simulations that the free energy of hydrophobic hydration is mainly determined by the hard-core effect of water and less by the hydrogen bonding of water molecules, and the effect of the latter could be seen in the coordination-number dependence of the average energy of one molecule. These facts suggest that a cavity-based model with the hard-core effect but without explicit inclusion of the hydrogen-bonding effect is useful as far as the hydration free energy is concerned, although the hydrogen bonding interactions are undoubtedly important in understanding molecular-level structures and dynamics of the mixtures.

In view of these situations, we estimate free energy of solvation using a cavity-based model. The solvation Gibbs energy as a Ben-Naim Standard quantity is given by, ${ }^{43,45}$

$$
\Delta G=\Delta G_{c}+\Delta G_{a},
$$

where $\Delta G_{c}$ denotes a energy of creating cavity for a solute molecule in a solvent, and $\Delta G_{a}$ a work of turning on the solute-solvent attractive potential. The former quantity is positive in nature and the latter negative. The Gibbs energy change on the transfer of a solute molecule (denoted by $s$ : $s=b$ for benzene and $s=w$ for water) from its neat liquid into a solvent is given by,

$\Delta \Delta G(s)=\Delta G^{\text {Solv }}(s)-\Delta G^{\text {Neat }}(s)=\Delta \Delta G_{c}(s)+\Delta \Delta G_{a}(s)$,

where

$$
\begin{aligned}
& \Delta \Delta G_{c}(s)=\Delta G_{c}^{\text {Solv }}(s)-\Delta G_{c}^{\text {Neat }}(s), \\
& \Delta \Delta G_{a}(s)=\Delta G_{a}^{\text {Solv }}(s)-\Delta G_{a}^{\text {Neat }}(s) .
\end{aligned}
$$

Various expressions have so far been proposed to estimate the cavity creation energy and the solute-solvent interaction energy. ${ }^{45}$ In the present paper, we employ a recent sophisticated expression by Matyushov and Ladanyi ${ }^{54}$ for the cavity creation energy:

$$
\begin{aligned}
\Delta G_{c}= & R T\left[\frac{3 \eta}{1-\eta}\left(\sigma_{2} / \sigma_{1}\right)\right. \\
& +\frac{3 \eta(2-\eta)(1+\eta)}{2(1-\eta)^{2}}\left(\sigma_{2} / \sigma_{1}\right)^{2} \\
& \left.+\frac{\eta\left(1+\eta+\eta^{2}-\eta^{3}\right)}{(1-\eta)^{3}}\left(\sigma_{2} / \sigma_{1}\right)^{3}-\ln (1-\eta)\right],
\end{aligned}
$$

where $R$ is the gas constant, $\sigma_{1}$ and $\sigma_{2}$ are the hard sphere diameters of the solvent and solute molecules, respectively, and $\eta$ is the volume packing density of the solvent given by

$$
\eta=\pi \sigma_{1}^{3} N_{A} / 6 v_{1},
$$

where $v_{1}$ is the molar volume of the solvent. The Gibbs energy change due to solute-solvent interaction, $\Delta G_{a}$, is simply replaced by purely dispersion energy, $E_{a}$. This is based on an assumption that the solvent reorganization associated with the process of turning on the attractive potential is an enthalpy-entropy compensating process. ${ }^{45,55,56}$ The dispersion energy is estimated by, ${ }^{57}$

$$
E_{a}=-(64 / 3) \eta R \epsilon_{12}\left(\sigma_{12} / \sigma_{1}\right)^{3},
$$

where $\sigma_{12}=\left(\sigma_{1}+\sigma_{2}\right) / 2, \epsilon_{12}=\left(\epsilon_{1} \epsilon_{2}\right)^{1 / 2}$, and $\epsilon_{1}$ and $\epsilon_{2}$ are the Lennard-Jones potential parameters for the solvent and solute, respectively. The molar volumes of water and benzene at various temperatures and pressures were obtained from literature densities, ${ }^{39,40}$ and the other parameter values used were $\sigma_{W}=2.87 \AA, \sigma_{B}=5.27 \AA,{ }^{45} \epsilon_{W} / k_{B}=100 \mathrm{~K}$, and $\epsilon_{B} / k_{B}=531 \mathrm{~K},{ }^{56}$ where $k_{B}$ is the Boltzmann constant.

The resulting values of $\Delta \Delta G_{c}$ and $\Delta E_{a}$ are plotted against temperature at several pressures in Fig. 5. The change in the cavity creation energy, $\Delta \Delta G_{c}$, is obviously larger for the benzene transfer than for the water transfer. This fact indicates that the cavity creation energy is larger in liquid water than in liquid benzene, and in addition, more energy is required to create a cavity for a larger molecule, benzene, than that for a smaller molecule, water. The change in the solute-solvent interaction energy, $\Delta E_{a}$, is smaller in magnitude than $\Delta \Delta G_{c}$. Nevertheless, it is larger for the benzene transfer than for the water transfer. It is worthwhile to notice that the effect of pressure on $\Delta \Delta G_{c}$ is opposite to that on $\Delta E_{a}$ for both the benzene transfer and the water transfer. In addition, the pressure effect on the both quantities for the benzene transfer is reverse of those for the water transfer. In the present model, the pressure effect is allowed for through the pressure-dependent change of the molar volumes. Therefore, the opposite direction of the pressure effect between the benzene transfer and the water transfer is caused by difference in compressibility between benzene and water. Average isothermal compressibilities of water and benzene were estimated from the literature densities. ${ }^{39,40}$ The values obtained for water and benzene are $4.5 \times 10^{-5} \mathrm{bar}^{-1}$ and 1.3 $\times 10^{-4}$ bar $^{-1}$, respectively, at $373 \mathrm{~K}(50-400$ bar) and 1.5 $\times 10^{-4}$ bar $^{-1}$ and $5.8 \times 10^{-4}$ bar $^{-1}$, respectively, at $548 \mathrm{~K}$ (100-400 bar). Obviously, the compressibility of benzene is significantly larger than that of water, and so the pressure 

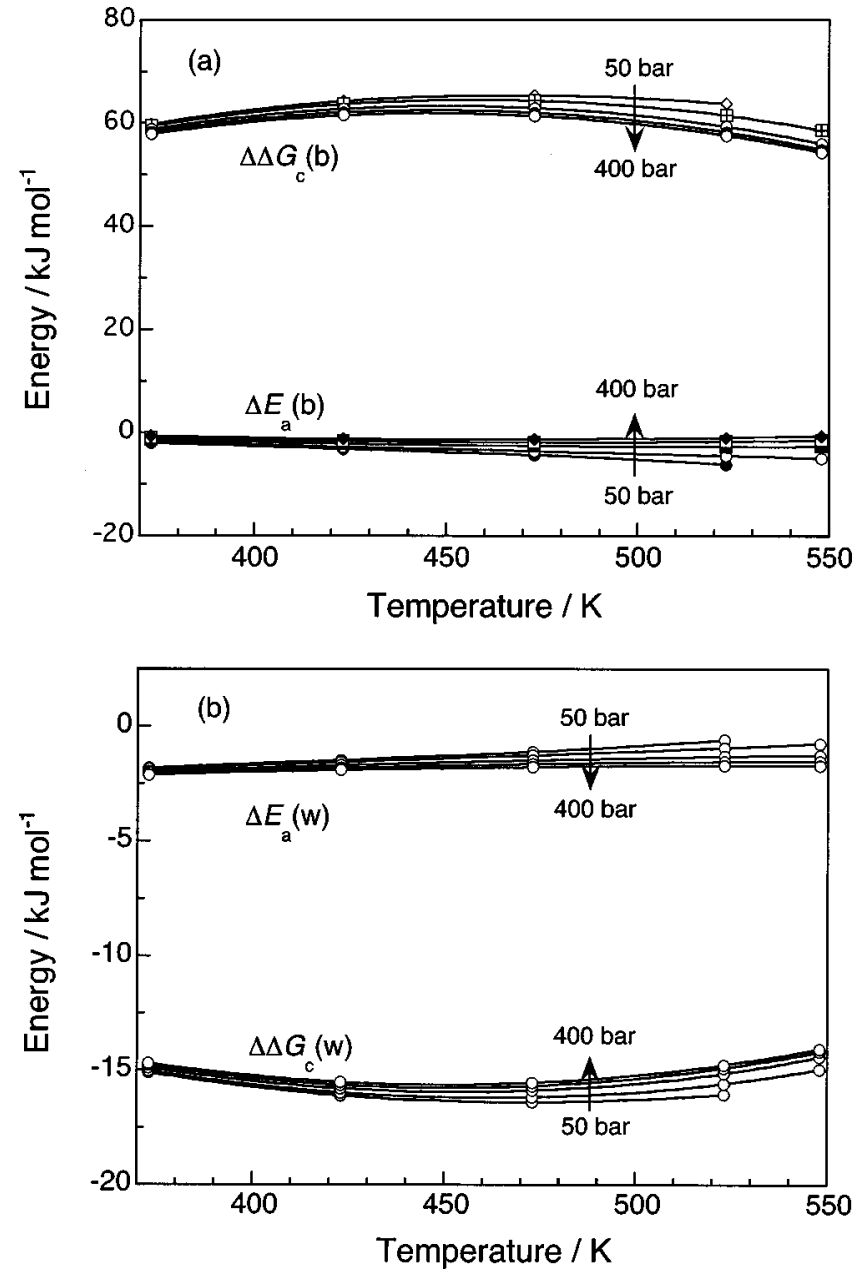

FIG. 5. Effect of temperature and pressure of the cavity creation energy change, $\Delta \Delta G_{c}$, and the solute-solvent interaction energy change, $\Delta E_{a}$, on transfer of (a) a benzene molecule from liquid benzene into liquid water and transfer of (b) a water molecule from liquid water into liquid benzene.

dependence of both the cavity creation energy and the solute-solvent interaction is larger in benzene than in water. Although the present model is for an infinite dilute solution, the results will be helpful to understand, at least qualitatively, the solubility properties of the mixtures.

Figure 6 shows plots of the Gibbs energy change for the molecular transfer, $\Delta \Delta G$, divided by thermal energy, $R T$, against temperature. The feature of its pressure-temperature dependence is mainly determined by that of the cavity creation energy change, $\Delta \Delta G_{c}$, the magnitude of which is distinctly larger than that of $\Delta E_{a}$. Since $-\Delta \Delta G / R T$ provides a measure of the solubility, the calculated results clearly indicate that dissolution of benzene in water is much harder than that of water in benzene. This is consistent with the asymmetry of the mutual solubility of water and benzene. In addition, the pressure dependence of $-\Delta \Delta G / R T$ shown in Fig. 6 agrees with the experimental fact that the solubility of benzene in water increases but that of water in benzene decreases with increasing pressure at constant temperature. Moreover, Fig. 6(a) clearly shows that the benzene solubility in water increases with increasing temperature, being consistent with the experimental results. On the other hand, the result in Fig. 6(b) cannot explain the experimental
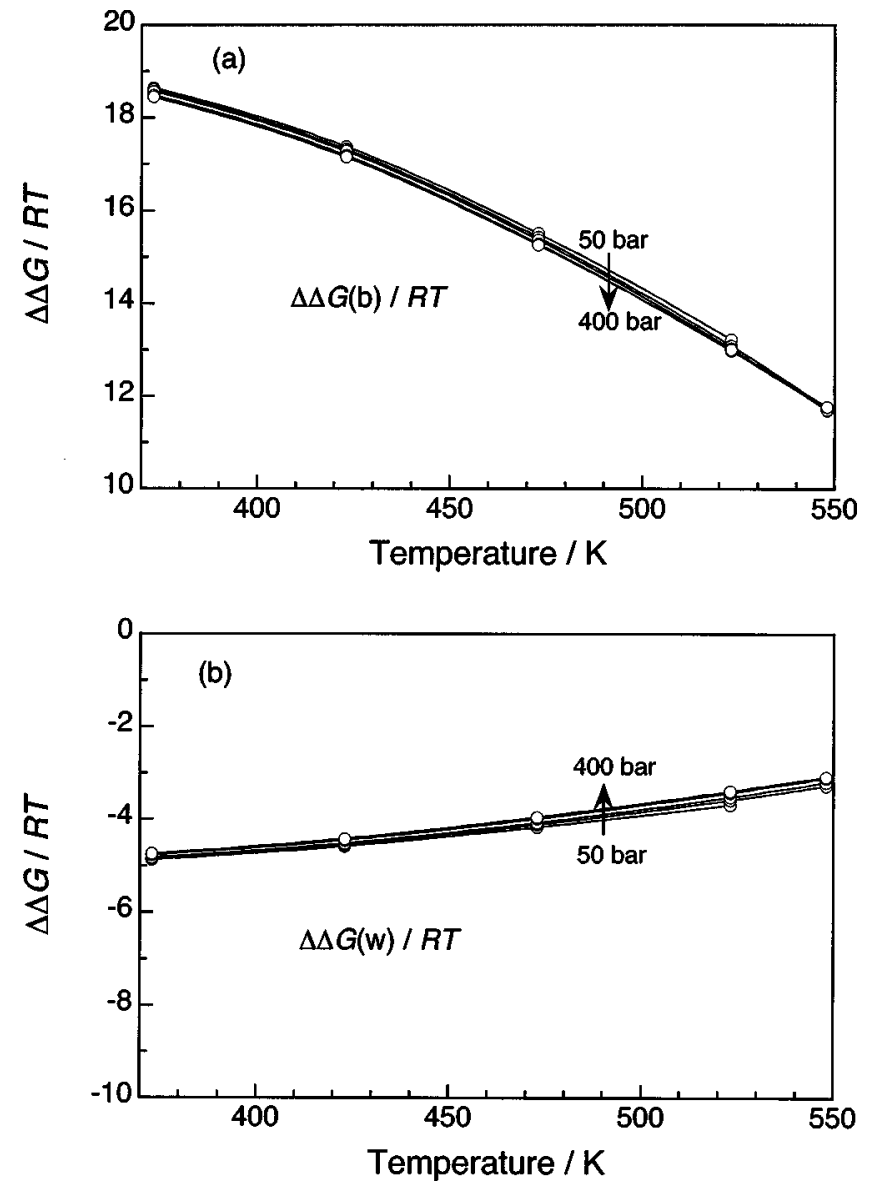

FIG. 6. Effect of temperature and pressure of the Gibbs energy change on transfer of (a) a benzene molecule from liquid benzene into liquid water and (b) a water molecule from liquid water into liquid benzene.

temperature-dependent increase of the water solubility in benzene. This fact may suggest that the free energy of the water transfer would be reduced further by water-water hydrogen-bonding interaction at finite dilution, which is not included in the present model for infinite dilution. This consideration is supported by the previous experimental result that a proportion of the hydrogen-bonded association of water in benzene increases as the water solubility in benzene increases with increasing temperature. ${ }^{58}$ This mixing effect caused by the water-water hydrogen-bonding interaction may yield negative larger value of $\Delta \Delta E_{a}(w)$, and then reduce $\Delta \Delta G(w)$ further at higher temperatures.

In conclusion, the asymmetry in the mutual solubilities of the water-benzene system can be at least qualitatively explained by the cavity-based model. The fact that the solubility of benzene in water is significantly smaller than that of water in benzene is explained by the considerable difference in molecular size between water and benzene, and the opposite direction of the pressure effect on the mutual solubilities can be understood by difference in isothermal compressibility between benzene and water.

\section{ACKNOWLEDGMENTS}

The authors thank Dr. Seiya Furutaka for the valuable discussions. They also thank Dr. Takuya Fukuda for his kind 
support in use of the near-infrared light source. This work was supported by the Grant-in-Aid for Scientific Research from the Ministry of Education, Culture, Sports, Science, and Technology of Japan (No. 13440169).

${ }^{1}$ G. A. Jeffrey and W. Saenger, Hydrogen Bonding in Biological Structures (Springer-Verlag, Berlin, 1991).

${ }^{2}$ T. E. Creighton, Proteins: Structure and Molecular Properties, 2 nd ed. (Freeman, New York, 1993).

${ }^{3}$ G. I. Makhatadze and P. L. Privalov, Adv. Protein Chem. 47, 307 (1995).

${ }^{4}$ J. Peng and A. Wan, Environ. Sci. Technol. 31, 2998 (1997).

${ }^{5}$ C. Tsonopoulos, Fluid Phase Equilib. 186, 185 (2001).

${ }^{6}$ Solubility Data Series, edited by A. L. Horvath and F. W. Getzen (Pergamon, Oxford, 1985), Vol. 20.

${ }^{7}$ Solubility Data Series, edited by D. G. Show (Pergamon, Oxford, 1989), Vol. 37.

${ }^{8}$ Solubility Data Series, edited by D. G. Show (Pergamon, Oxford, 1989), Vol. 38.

${ }^{9}$ C. Tsonopoulos and G. M. Wilson, AIChE J. 29, 990 (1983).

${ }^{10}$ F. E. Anderson and J. M. Prasnitz, Fluid Phase Equilib. 32, 63 (1986)

${ }^{11}$ D. J. Miller and S. B. Hawthorne, J. Chem. Eng. Data 45, 78 (2000).

${ }^{12}$ C. Marche, H. Delepine, C. Ferronato, and J. Jose, J. Chem. Eng. Data 48, 398 (2003)

${ }^{13}$ F.-Y. Jou and A. E. Mather, J. Chem. Eng. Data 48, 750 (2003).

${ }^{14}$ T. Sako, T. Sugeta, K. Otake, M. Sato, M. Tsugumi, T. Hiaki, and M. Hongo, J. Chem. Eng. Jpn. 30, 744 (1997).

${ }^{15}$ J. W. Tester and J. A. Cline, Corrosion (Houston) 55, 1088 (1999).

${ }^{16}$ Y. Yamasaki, H. Enomoto, N. Yamasaki, and M. Nakahara, Bull. Chem. Soc. Jpn. 73, 2687 (2000)

${ }^{17}$ P. Kritzer and E. Dinjus, Chem. Eng. J. 83, 207 (2001).

${ }^{18}$ J. S. Rowlinson and F. L. Swinton, Liquids and Liquid Mixtures, 3rd ed. (Butterworth, London, 1982).

${ }^{19}$ C. J. Wormald, Ber. Bunsenges. Phys. Chem. 88, 826 (1984).

${ }^{20} \mathrm{~J}$. Li, I. Vanderbeken, S. Ye, H. Carrier, and P. Xans, Fluid Phase Equilib. 131, 107 (1997)

${ }^{21}$ K. Chandler, B. Eason, C. L. Liotta, and C. A. Eckert, Ind. Eng. Chem. Res. 37, 3515 (1997).

${ }^{22}$ W. H. Thompson and J. R. Snyder, J. Chem. Eng. Data 9, 516 (1964).

${ }^{23}$ J. F. Connolly, J. Chem. Eng. Data 11, 13 (1966).

${ }^{24}$ R. G. Sultanov and V. G. Skripka, Zh. Fiz. Khim. 47, 1035 (1973); V. G. Skripka, Tr. Vses. Neftegazov. Nauchno-Issled. Inst. 61, 139 (1976).

${ }^{25}$ G. T. Hefter and C. L. Young, in Solubility Data Series, edited by D. G. Show (Pergamon, Oxford, 1989), Vol. 37
${ }^{26}$ S. Furutaka and S. Ikawa, J. Chem. Phys. 113, 1942 (2000).

${ }^{27}$ Y. Jin and S. Ikawa, J. Chem. Phys. 119, 12432 (2003).

${ }^{28}$ T. Tassaing, Vib. Spectrosc. 24, 15 (2000).

${ }^{29}$ T. Tassaing, Y. Dantan, and M. Besnard, J. Mol. Liq. 101, 149 (2002).

${ }^{30}$ R. Oparin, T. Tassaing, Y. Danten, and M. Besnard, J. Chem. Phys. 120, 10691 (2004)

${ }^{31}$ S. Furutaka and S. Ikawa, J. Chem. Phys. 117, 751 (2002).

${ }^{32}$ S. Furutaka and S. Ikawa, J. Chem. Phys. 117, 1682 (2002).

${ }^{33}$ Y. Jin and S. Ikawa, J. Chem. Phys. 121, 2694 (2004).

${ }^{34}$ R. S. Bradley, M. J. Dew, and D. C. Munro, High Temp. - High Press. 5, 169 (1973).

${ }^{35}$ S. Sawamura, K. Kitamura, and Y. Taniguchi, J. Phys. Chem. 93, 4931 (1989).

${ }^{36}$ J. H. Callomon, J. M. Dunn, and I. M. Mills, Philos. Trans. R. Soc. London, Ser. A 259, 499 (1966).

${ }^{37}$ J. I. Steinfeld, Molecules and Radiation (MIT Press, Cambridge, 1985).

${ }^{38}$ Corrected form of Eq. (1) of Ref. 33 which lacked enough significant figures.

${ }^{39}$ E. W. Lemmon, M. O. McLinden, and D. G. Friend, in NIST Chemistry WebBook, NIST Standard Reference Database Number 69, edited by P. J. Linstrom and W. G. Mallard (National Institute of Standards and Technology, Gaithersburg, MD, 2001) (http://webbook.nist.gov/).

${ }^{40}$ R. D. Goodwin, J. Phys. Chem. Ref. Data 17, 1541 (1988).

${ }^{41}$ J. Polak and B. C.-Y. Lu, Can. J. Chem. 51, 4018 (1973).

${ }^{42}$ A. V. Plyasunov and E. L. Shock, Geochim. Cosmochim. Acta 64, 2811 (2000).

${ }^{43}$ A. Ben-Naim, Hydrophobic Interactions (Plenum, New York, 1980).

${ }^{44}$ J. L. Finney, Faraday Discuss. 103, 1 (1996).

${ }^{45}$ R. Schmid, Monatsch. Chem. 132, 1295 (2001).

${ }^{46}$ G. I. Makhadze and P. L. Privalov, Biophys. Chem. 50, 285 (1994).

${ }^{47}$ M. Costas and B. Kronberg, Biophys. Chem. 74, 83 (1998).

${ }^{48}$ S. Suzuki, P. G. Green, R. E. Bumgarner, S. Dasgupta, W. A. Goddard III, and G. A. Blake, Science 257, 942 (1992).

${ }^{49}$ R. N. Pribble and T. S. Zwier, Faraday Discuss. 97, 229 (1994).

${ }^{50}$ A. Engdahl and B. Nelander, J. Phys. Chem. 91, 2253 (1987).

${ }^{51}$ M. Nakahara and C. Wakai, Chem. Lett. 1992, 809 ().

${ }^{52}$ G. Graziano, Biophys. Chem. 110, 249 (2004).

${ }^{53}$ M. Ikeguchi, S. Shimizu, S. Nakamura, and K. Shimizu, J. Phys. Chem. B 102, 5891 (1998).

${ }^{54}$ D. V. Matyushov and B. M. Ladanyi, J. Chem. Phys. 107, 5815 (1997).

${ }^{55}$ B. Lee, Biopolymers 24, 813 (1985); 31, 993 (1991).

${ }^{56}$ G. Graziano, Can. J. Chem. 80, 401 (2002).

${ }^{57}$ R. A. Pierotti, Chem. Rev. (Washington, D.C.) 76, 717 (1976).

${ }^{58}$ S. Furutaka and S. Ikawa, J. Chem. Phys. 108, 1347 (1998). 